Echenne, Service de neuropediatrie, Departemente de pediatrie, Universite de Sherbrooke, 3001, 12e Avenue Nord, CHUS, Fleurimont, Quebec, J1H 5N4, Canada).

COMMENT. Sepiapterin reductase deficiency, an autosomal recessive, doparesponsive, neurotransmitter disease, presents as an infantile encephalopathy between 2 and 6 months of age with delayed developmental milestones, hypotonia, later replaced by spasticity, oculogyric crises, and dystonic movements, with diurnal variation of motor abnormalities. Diagnosis, often mislabeled as cerebral palsy, is confirmed by CSF examination of neurotransmitters. Treatment consists of levodopa, carbidopa and 5-OH tryptophan. Response is dramatic with improvement in motor function and control of oculogyric crises, but persistence of moderately impaired cognitive function and learning. Neville BGR and colleagues at Great Ormond Street Hospital, London, previously reported 7 cases of SRD from Malta, with similar findings and response to therapy (Brain 2005;128:2291-2296; Ped Neur Briefs Oct 2005;19:78). The clinical manifestations of SRD resemble the autosomal dominant dopa responsive, Segawa disease (Ann Neurol 2003;54(Suppl 6):S32-S45). Infants with unexplained cerebral palsy should be screened for $\mathrm{SRD}$, and patients with action dystonia and/or oculogyric crises should receive a trial of Ldopa.

\title{
JUVENILE GM2 GANGLIOSIDOSES
}

Clinical features and genetic correlations of 21 new case histories and 134 published case reports of juvenile or subacute GM2 gangliosidosis were analyzed to delineate the natural history of the disorder, in a study at the Hospital for Sick Children, Toronto, Canada, and University of Sao Paulo, Brazil. Fifteen of the 21 patients had the Tay-Sachs variant and 6 the Sandhoff variant. The mean age of onset of symptoms was $5.3+/-4.1$ years, and the mean follow-up was 8.4 years. Presenting symptoms were gait disturbance in $67 \%$, incoordination $(52 \%)$, speech problems $(27 \%)$, and developmental delay $(29 \%)$. Patients became wheelchair-bound after $6.2+/-5.5$ years. Late signs included muscle wasting at 10.6 +/- 7.4 years, proximal weakness $(11.1+/-7.7$ years), and incontinence $(14.6+/-9.7$ years). Psychiatric disorders and neuropathy were more typical of the Sandhoff variant, and dysphagia, sphincter incontinence, and sleep problems presented earlier in the Tay-Sachs variant. MRI showed cerebellar atrophy in 53\%. Symptoms in the 21 new cases were similar to the 134 previous reports. Median survival of the total group of 155 patients was 14.5 years. Presence of $\mathrm{R} 178 \mathrm{H}$ and $\mathrm{R} 499 \mathrm{H}$ mutations was predictive of early onset and rapid progressive course. G269S or W474C mutations correlated with a later onset and slower progression. HEXA genotype in Tay-Sachs variant cases significantly correlated with clinical course. In both variants, speech deteriorated more rapidly than gait abnormalities. (Maegawa GHB, Stockley T, Tropak M et al. The natural history of juvenile or subacute GM2 gangliosidosis: 21 new cases and literature review of 134 previously reported. Pediatrics November 2006;118:1550-1562). (Respond: Joe TR Clarke MD PhD, Division of Clinical and Metabolic Genetics, Hospital for Sick Children, 555 University Ave, Toronto, Ont, Canada M5G 1X8).

COMMENT. Juvenile GM2 gangliosidosis is heterogeneous in age of onset, clinical features, and course. The earlier the onset, the more rapid the progression. 